\title{
Research on the Ground Pressure Features of Long Wall Top Coal Caving Working Face in Extra-Thick Coal Seam
}

\author{
Ma Zhanyuan ${ }^{1}$, Liu Chuang ${ }^{1,2}$, Li Huamin ${ }^{3,}$, \\ ${ }^{1}$ Jinneng Holding Group, Datong, China \\ ${ }^{2}$ School of Resource and Safety Engineering, Henan University of Engineering, Zhengzhou, China \\ ${ }^{3}$ School of Energy Science and Engineering, Henan Polytechnic University, Jiaozuo, China \\ Email address: \\ Ma781128@Chinaren.Com (Ma Zhanyuan), liuchuangyouxiang@163.com (Liu Chuang), lihm1957@163.com (Li Huamin) \\ ${ }^{*}$ Corresponding author
}

\section{To cite this article:}

Ma Zhanyuan, Liu Chuang, Li Huamin. Research on the Ground Pressure Features of Long Wall Top Coal Caving Working Face in ExtraThick Coal Seam. International Journal of Oil, Gas and Coal Engineering. Vol. 9, No. 2, 2021, pp. 12-16. doi: 10.11648/j.ogce.20210902.11

Received: March 24, 2021; Accepted: April 6, 2021; Published: May 21, 2021

\begin{abstract}
Based on the raw data of shield pressure and overlying strata movement measured in the field, the ground pressure features and overlying strata movement were analyzed in 8202 extra-thick coal seam longwall top coal caving working face of Tongxin coal mine after its extraction. The research results show that: during the process of mining, the interval of the lower sub key strata caved for the first time is $120 \mathrm{~m}$, the periodic weighting interval ranges from $15 \mathrm{~m}$ to $30 \mathrm{~m}$, with an average of $25 \mathrm{~m}$. When the immediate roof is relatively thick, the fractured lower sub key strata can be hinged to form a stable "Voussoir Beam" structure, which makes the ground pressure not severe and the shield pressure small in the ends of the panel. When the immediate roof is thin, the arc triangular of the lower sub key strata in the ends of the panel loses mechanical contact to the caved immediate roof and assumes a "Cantilever Beam" structure, which makes the ground pressure severe and shield pressure large in the ends of the panel. Due to the large mining space of longwall top coal caving working face in extra-thick coal seam and big rotary angle of the overlying strata, the lower sub key strata can't connect with each other to form a stable bearing structure, therefore, the shield pressure is a given load of roof rock, which results in a constant pressure of the shield.
\end{abstract}

Keywords: Extra-thick Coal Seam, Top Coal Caving Face, Strata Movement, Ground Pressure Features, Shield Pressure

\section{Introduction}

With the development of China's coal mining technology, mine construction and coal mining toward large-scale, intensive direction of development trend, China's extra-thick coal seam mining is an urgent need for high yield and efficiency of coal mining methods [1-4]. Compared with the traditional layered mining caving technology, longwall top coal caving (LTCC) is a good method to exploit the extrathick coal seam, with the high yield and high efficiency, low cost of roadway excavation rate, low cost of raw coal, strong adaptability and high safety [5-9]. Now, LTCC has been rapidly gaining popularity after it was introduced to Chinese nearly 30 years ago.

Most researches in China are concentrated in the aspects of working surface equipment, ground pressure control and mining method selection on the extra-thick coal seam.
However, there are few studies on the mutual influence of dual coal seams mining in extra-thick coal seam. Two main coal seams in Tongxin coal mine (Figure 1: Location of Tongxin coal mine), upper coal seam is the Jurassic, has been mined and golf tends to be stable. The distance between the two coal seam layers is $150 \mathrm{~m}$. In the process of the lower coal seam mining, the overlying strata are affected by the mining action, the stability of surrounding rock is poor, the movement feature of overlying rock is irregular, and the randomness is strong, which brings hidden danger to the safety production of mine. Based on this, taking Tongxin coal mine 8202 LTCC working face as an example, research on extra-thick coal seam under gob caving roof strata movement and ground pressure features, in order to achieve the purpose of safe and efficient mining working face, provide a reference for the similar conditions of coal mining. 


\section{General Situation of Engineering}

8202 LTCC working face is mining 3-5\# Carboniferous coal seam. Mining height of 8202 working face ranges from $13.61 \mathrm{~m}$ to $28.92 \mathrm{~m}$, with an average of $20.58 \mathrm{~m}$. The $3-5 \#$ coal seam is stable and consisting of 8-15 layers, among them, with $1.96 \mathrm{~m}$ gangue, accounting for $9.6 \%$. Polodyakonov coefficient of 3-5\# coal seam is $1-2$, resulting in an easy collapsing of top coal. 8202 working face is using comprehensive mechanized, low top coal caving, longwall retreating mining, multi round and hierarchical sequential caving method, natural collapse method for the overlying roof, with a $0.8 \mathrm{~m}$ cycle progress and coal caving step distance, until the working face advances to $30 \mathrm{~m}$ before the mining stop line. 8202 top coal caving face is only mining the cutting height, no caving top coal, between the stop mining line and $30 \mathrm{~m}$ before. The layout of gate roads of 8202 top coal caving face is shown in figure 2 .

The thickness of the lower sub key strata is $17.04 \mathrm{~m}$, which consists of sandy mudstone, No. 1 coal seam, coarse sandstone and fine sandstone. Immediate roof thickness is $3.87 \mathrm{~m}$, for Carbon Mudstone and 2\# coal seam. Immediate bottom is sandy mudstone, $1.98 \mathrm{~m}$ thick, grayish brown, containing carbonaceous clip and kaolin coal line. Main floor is sandy mudstone, dark gray, thick layer.

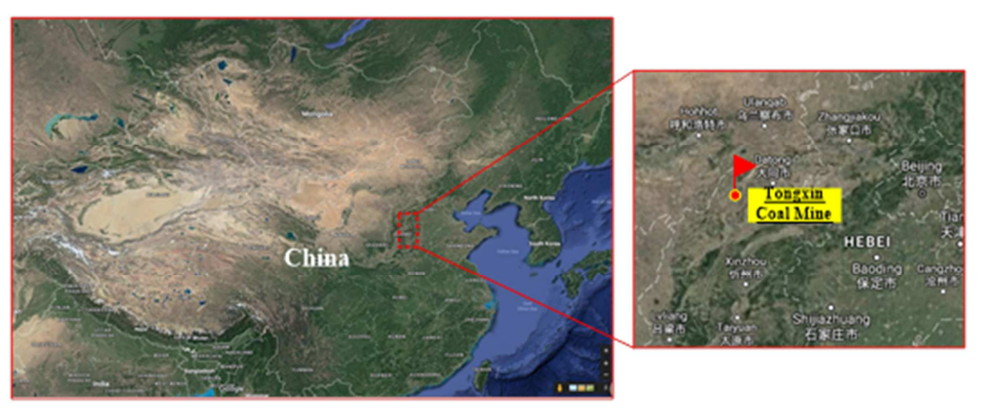

Figure 1. Location of Tongxin coal mine.

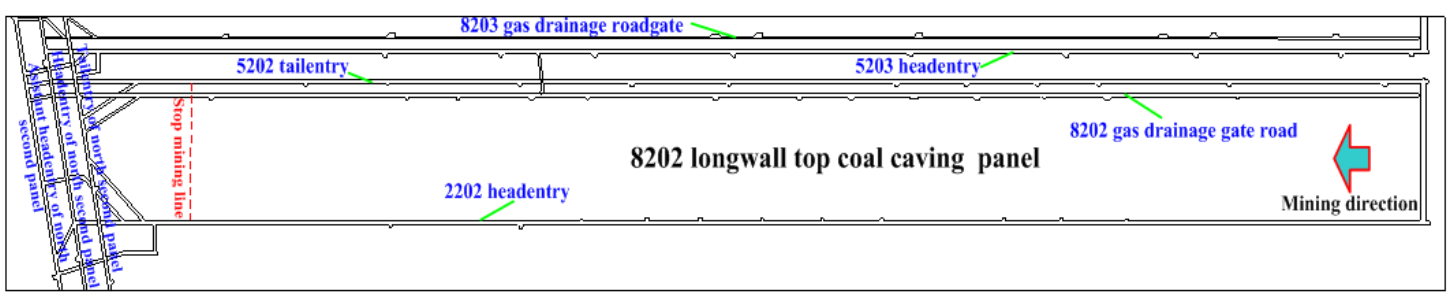

Figure 2. Gate roads layout of 8202 LTCC panel.

The ZF15000/27.5/42 low top coal caving type shield is selected for the 8202 LTCC working face. The support height of this type shield is $2.75-4.20 \mathrm{~m}$, the center-to-center distance between adjacent shields of $1.75 \mathrm{~m}$, the yield pressure of 36.86 $\mathrm{MPa}(15000 \mathrm{kN})$, and the setting pressure of $31.4 \mathrm{MPa}(12778$ $\mathrm{kN})$. In actual applications, due to the supply pipe pressure and other factors, the yield and setting pressure of the shield are $31.0 \mathrm{MPa}(12615 \mathrm{kN})$ and 14.0 MPa $(5700 \mathrm{kN})$, respectively. In order to adapt to the shields in the middle of the working face, the ZFG13000/27.5/42 H low caving top coal type is selected as transition shield and ZTZ20000/30/42 as end position shield. The working face is arranged in a total of 108 middle shields, 9 transition shields (4 transition shields arranged in the upper working face, and 5 transition shields arranged in the lower working face). The upper of the working face is arranged with 1 sets of end shield, including 2 shields.

\section{Ground Pressure Observation}

11 sets of KJ21 online ground pressure monitoring system recorders are installed in the middle working face shields of 8202 LTCC working face, that is, one measuring point is arranged every ten shields, and the monitoring points are arranged as shown in figure 3. The monitoring system records a data every 5 minutes, real-time record of the changes of the shield pressure during its process of rising, moving and falling. The monitoring date is from 13 August to 9 October 2016, during which a total of $278 \mathrm{~m}$ of working face advances.

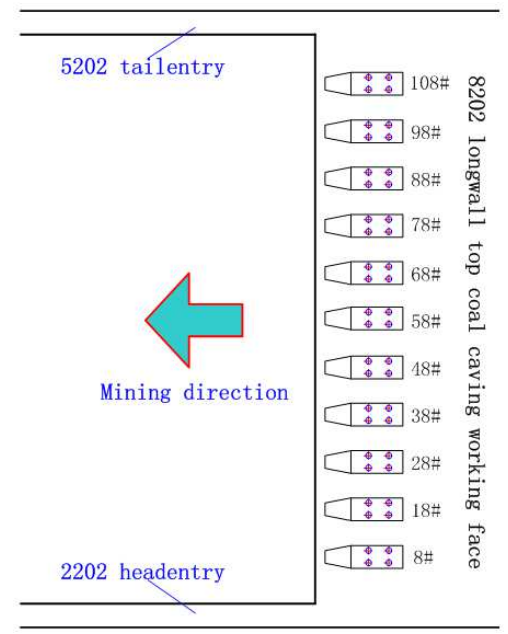

Figure 3. Layout of shield pressure monitoring station. 


\subsection{Features of Overlying Strata Periodic Weighting}

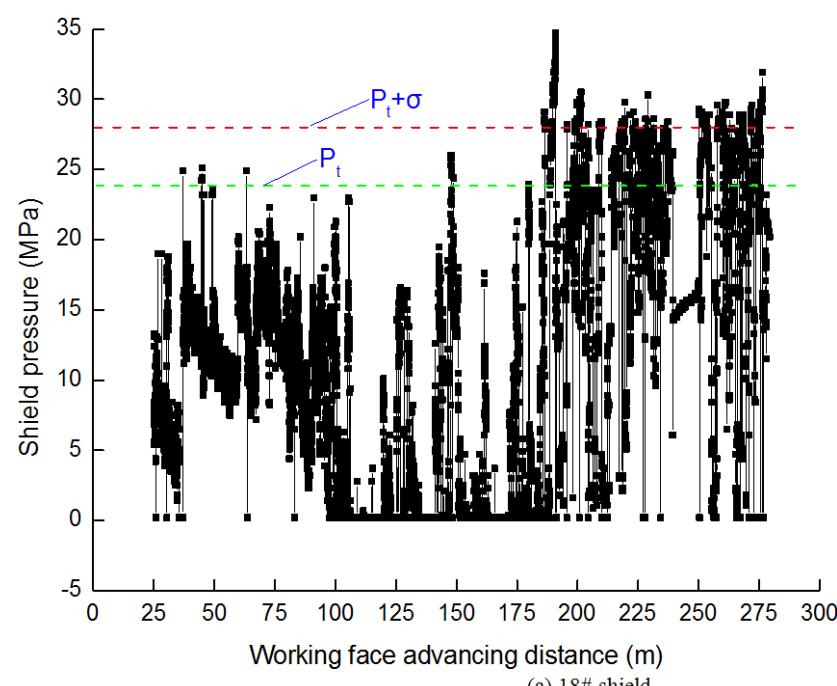

(a) $18 \#$ shield

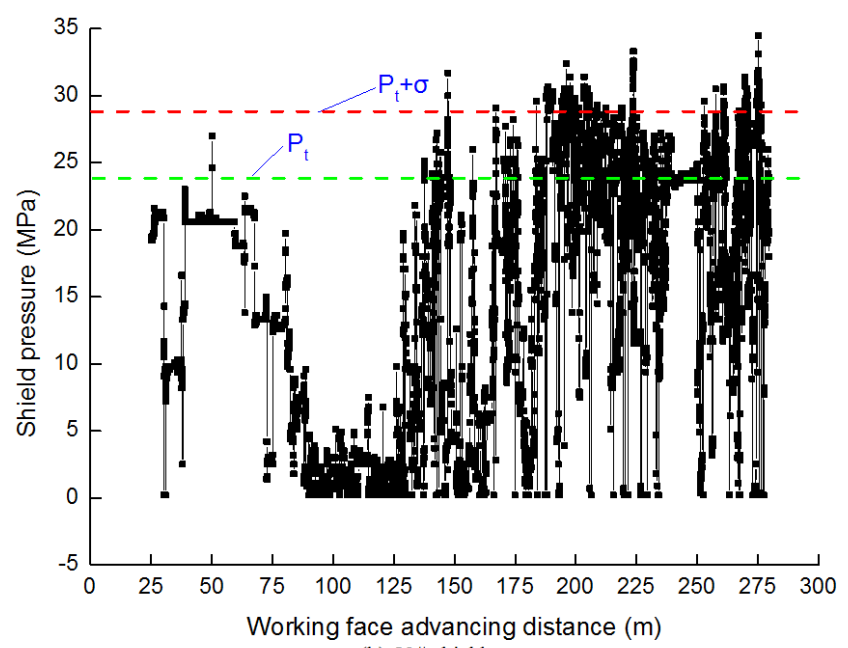

(b) 58\# shield

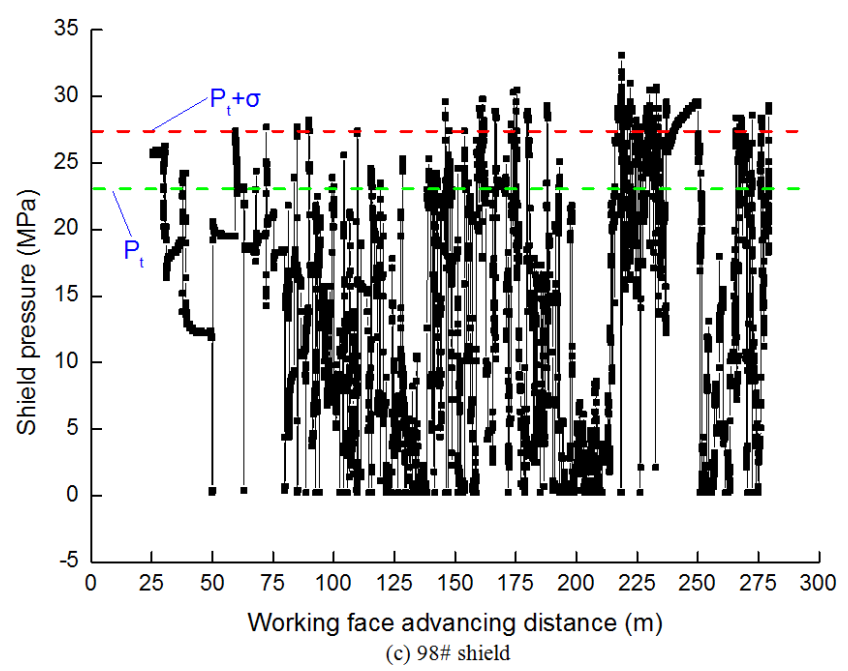

Figure 4. Changes of shield pressure as the face advances.

The sum of the average of shield pressure at the end of a cycle $\mathrm{P}_{t}$ and its square deviation $\sigma\left(\mathrm{P}_{t}+\sigma\right)$ is taken as the main index to judge the cycle pressure of the overlying roof strata. According to the calculation results of the periodic weighting criterion, the middle period of working face is higher than the two ends of the working face.

The periodic weighting criterion of $48 \#$ shield is maximum, $30.71 \mathrm{MPa}$. $88 \#$ shield is $25.73 \mathrm{MPa}$, which is only $70 \%$ of the yield working pressure and indicates that the overlying strata strength around the $88 \#$ shield is weak and the roof pressure appears to ease. The periodic weighting criterion is obtained after the calculation of the average of shield pressure at the end of a cycle $P_{t}$ and its square deviation $\sigma$. 18\#, 58 \# and 98 \# shield in the upper, middle and lower working face are selected to analyze the working pressure of each shield.

As shown in figure 4, according to the raw data of $18 \#$, $58 \#, 98 \#$ shield pressure during the $25-278 \mathrm{~m}$ mining period, the overlying roof strata of the working face is periodic caving. The lower sub key strata initial weighting distance is 100-140 m, with an average of $120 \mathrm{~m}$, meanwhile the periodic weighting distance is $15 \sim 30 \mathrm{~m}$, with an average of $25 \mathrm{~m}$. The periodic weighting characteristics is not obvious, and shield pressure slightly increases during the periodic weighting time.

\subsection{Shield Pressure Features Along the Working Face Direction}

Figure 5 shows that the square deviation of the average of $38 \#-68 \#$ shield pressure at the end of a cycle is large, indicating that the ground pressure around the range appears more strongly.

The square deviation of the average of $38 \#$ shield pressure at the end of a cycle is the maximum at $5.87 \mathrm{MPa}$, and the $108 \#$ shield is the least, which is $3.47 \mathrm{MPa}$. In the whole working face, the square deviation of the average of $38 \#$ shield pressure at the end of a cycle presents a big trend in the middle and both ends, indicating that the ground pressure in the middle of the working face is higher than the two ends.

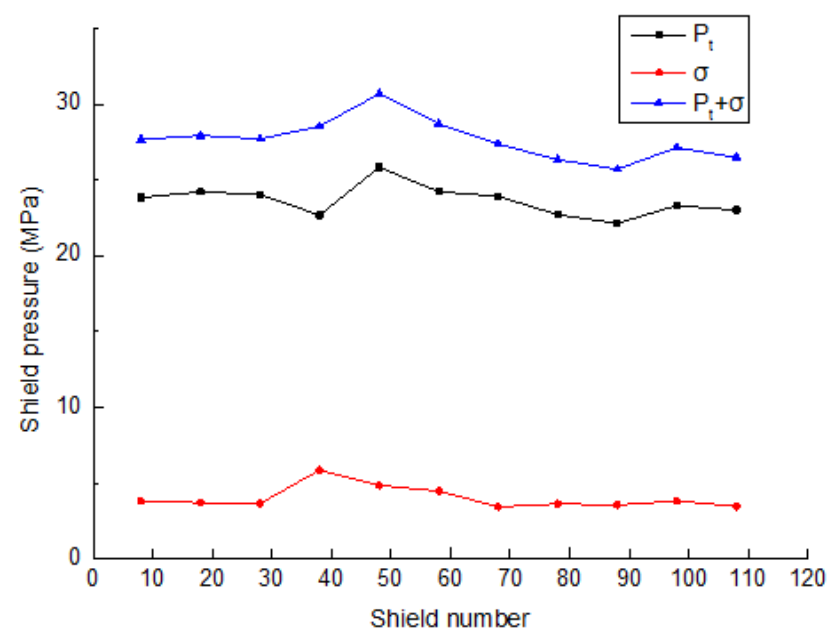

Figure 5. Curve of average of shield pressure at the end of a cycle and its square deviation.

\section{Analysis of Shield Pressure}

From the shield pressure curve can be seen, the working 
pressure of shield in the mining period changes little (without considering the shield maintenance and falling stage) relative to the working face with large mining height. That's because the mining space of the top coal caving face is not consistent with the support space, as the transfer medium to apply the load directly to the shield is top coal rather than rock [10-14]. The density of coal is small, and the compression is brittle and loose, the joint and crack is much more than that of rock, the fluidity is big, the overall stability is bad. After being discharged and recovered, the top coal has enough free space in the mined-out area. Under the support pressure, the top coal body is compressed in the longitudinal direction and expands and breaks in the lateral direction [15]. During the process of the working face mining, the energy is gradually released to the mined-out area, and the top coal is broken and crushed.

Due to the recovery of top coal in top coal caving face, the space needed to fill in the gob is increased exponentially, and only the higher full caving zone can maintain the balance of the whole stope rock mass. In the case of extra-thick immediate roof, the collapse height of caving zone is increased; when the thickness of the immediate roof is not sufficient, the upper overlying strata with a certain thickness will be used as the rock block of the regular collapse zone to compensate for the lack of gob filling. Due to the large rotation angle of the lower sub key strata, resulting in the horizontal projection length of the rock beam is shortened greatly. When the change of the contact position between the rock beams is not enough to compensate for the shortened displacement, rock beam loses the lateral force of contact. The lower sub key strata above this layer is equivalent to the fractured zone strata of slicing mining. Although it can form a balanced structure of "Voussior Beam", but due to the formation of a long time, the formation has been far away from the mining face, it doesn't constitute a big impact on the mining space [16-18].

Therefore, in different geological conditions of top coal caving faces, shield pressure is small and has little difference, and no significant increase or decrease in pressure before and after the periodic weighting, the reason is that the external load of the shield is a given load which is the function of top coal and the caving zone that can collapse.

\section{Conclusion}

(1) The initial weighting distance of lower sub key strata is $120 \mathrm{~m}$, the periodic weighting interval is $25 \mathrm{~m}$ in Tongxin 8202 LTCC working face.

(2) The work pressure of shield in middle section of top coal caving face is higher than the two ends, and the ground pressure of middle shield is stronger than that of the two ends.

(3) For the extra-thick top coal caving face, the working pressure of shield is a given load, the working pressure of shield during mining changes relatively little.

(4) When the immediate roof is thin, the arc triangular of the lower sub key strata in the ends of the panel loses mechanical contact to the caved immediate roof and assumes a "Cantilever Beam" structure, which makes the ground pressure severe and shield pressure large in the ends of the panel. When the immediate roof is relatively thick, the fractured lower sub key strata can be hinged to form a stable "Voussoir Beam" structure, which makes the ground pressure not severe and the shield pressure small in the ends of the panel.

\section{Acknowledgements}

This research is funded by the National key R\&D Program of China 2018YFC0604502. The authors would like to thank Datong Coal Mine Group Co., Ltd. Tongxin and Tashan Coal Mine for financial support.

\section{References}

[1] YU L., YAN S. H. (2020). The basic principle of roof strata control in fully mechanized caving mining of extra thick coal seam. Journal of China Coal Society, 45 (S1): 31-37. doi: 10.13225/j.cnki.jccs.2020.0230.

[2] REN Q. H., XU Z. Y., CHEN C. (2021). Overburden structure and rock pressure law of fully-mechanized top coal caving stope in extra-thick coal seam. Coal Engineering. 53 (01): 7983.

[3] Li H. M., JIANG D. J., Syd S. P. (2015). Analysis on loading features and suitability of hydraulic powered caving supports. Coal Science and Technology. 43 (6): 22-28, 70.

[4] Yu B., ZHU W. B., GAO R. (2016). Strata structure and its effect mechanism of large space stope for fully-mechanized sublevel caving mining of extremely thick coal seam. Journal of China Coal Society. 41 (3): 571-580.

[5] KONG L. H. (2020). Overlying strata movement law and its strata pressure mechanism in fully mechanized top-coal caving workface with large space. Journal of Mining \& Safety Engineering. 37 (05): 943-950. doi 10.13545/j.cnki.jmse. 2020.05.010.

[6] WANG H. J., LIU Y. J. (2020). Study on overlying strata movement and stress dynamic evolution above working face in $8.8 \mathrm{~m}$ extra-thick coal seam. Coal Science and Technology, 48 (11): 68-76. doi: 10. 13199 /j. cnki.cst.2020.11.009.

[7] Huang Z. Z. (2013). Study on laws of roof strata fracture of fully mechanized caving mining under goaf in ultra-thick seam. Coal Science and Technology. 41 (7): 60-62, 66.

[8] Yan S. H. (2009) Theory study on the load on support of longwall with top coal caving with great mining height in extra thick coal seam. Journal of China Coal Society. 34 (5): 590-693.

[9] Jia X. R., ZHAI Y. D., YANG S. S. (1998). Structure of roof rocks in sub-level caving face and calculation of rock weighting. Journal of China Coal Society. 23 (4): 366-370.

[10] LIU C., LI H. M., Mitri Hani, JIANG D. J., CHEN W. X. (2020). Strata movement analysis at Tongxin longwall top coal caving working face with extra-thick coal seam. Arabian Journal of Geosciences, doi: 10.1007/s12517-019-4787-0. 
[11] LIANG S. P., LU Y. L., GUO P., WU B. Z. (2020). Mechanical Analysis of the First Fracture Characteristics of Hard Roof of Extra-thick Coal Seam. Safety in Coal Mines, 51 (8): $245-250$.

[12] LIU C., LI H. M., Mitri Hani (2017). Voussoir beam model for lower strong roof strata movement in longwall mining-Case study. Journal of Rock Mechanics and Geological Engineering, 9 (6): 1171-1176.

[13] Zhu S. S., LI H. C., YANG Z. F. (1996). The structure of overlying coal and rocks in sublevel caving faces. Chinese Journal of Mechanics and Engineering. 15 (2): 150-154.

[14] Gu Q. Z., SHI Y. W., QI Q. X. (1996). Rules of roof movement in sub-level caving workings. Journal of China Coal Society. 21 (1): 45-50.

[15] Kang T. H., CHAI Z. Y., LI Y. B. (2007). Study on physical simulation of full-seam mining for a $20 \mathrm{~m}$ very thick and medium hard seam by sub-level caving mining with high bottom cutting height. Chinese Journal of Rock Mechanics and Engineering. 26 (5): 1065-1072.

[16] LIU C., LI H. M. \& Mitri Hani (2019). Effect of Strata Conditions on Shield Pressure and Surface Subsidence at a Longwall Top Coal Caving Working Face. Rock Mechanics and Rock Engineering, 52: 1523-1537.

[17] Li H. M., JIANG D. J., LI D. Y. (2014) Analysis of ground pressure and roof movement in fully-mechanized top coal caving with large height in ultra-thick seam. Journal of China Coal Society, 39 (10): 1956-1960.

[18] Yu B., LIU C. Y., YANG J. X. (2014). Mechanism of strong pressure reveal under the influence of mining dual system of coal pillar in Datong mining area. Journal of China Coal Society. 39 (1): 40-46. 